\title{
Aspectos conceituais e metodológicos da resiliência psicológica: uma análise da produção científica brasileira
}

\section{Conceptual and methodological aspects of psychological resilience: an analysis of Brazilian scientific production}

\section{Aspectos conceptuales y metodológicos de la resiliencia psicológica: una análisis de la producción científica brasileña}

\author{
Mayse Itagiba Rooke*
}

Universidade Federal de Juiz de Fora - UFJF, Juiz de Fora, Minas Gerais, Brasil

\begin{abstract}
RESUMO
Estudos acerca da resiliência psicológica têm sido frequentes na produção científica brasileira. Em geral, as discussões a respeito deste construto salientam os processos de adaptação positiva como respostas no enfrentamento de adversidades significativas. Assim, o presente estudo busca apresentar um panorama acerca da produção científica sobre a resiliência psicológica no âmbito nacional, a partir da análise de artigos indexados nas bases de dados Lilacs e SciELO no período de 1970 a 2014. Foram encontrados 89 artigos, sendo 47 trabalhos empíricos e 42 pesquisas teóricas, a maioria com foco na resiliência individual $(n=75 ; 84,3 \%)$. Além disso, observou-se que os estudos adotam, frequentemente, métodos qualitativos, especificamente, delineamentos transversais e descritivos com poucos participantes, bem como utilizam, geralmente, apenas um instrumento na coleta de dados: a entrevista. No que tange aos assuntos investigados, a maioria dos trabalhos é referente à 'resiliência e adversidades' $(n=38 ; 42,7 \%)$. Verifica-se que a resiliência carece de uma definição consensual e uma tradição empírica consolidada, enfatizando a importância de trabalhos como este para os pesquisadores da área.
\end{abstract}

Palavras-chave: resiliência psicológica, pesquisa científica.

\begin{abstract}
Studies on the psychological resilience are frequent in the Brazilian scientific production. In general, discussions about this construct enphasizes the positive adaptation processes as responses facing significant adversity. This study aims to present an overview of scientific studies on the psychological resilience at the national level based on the analysis of articles indexed in the Lilacs and SciELO databases from 1970 to 2014. Were found 89 articles, 47 empirical studies and 42 theoretical research, many of them focusing on individual resilience $(n=75 ; 84,3 \%)$. In addition, it was observed that the studies uses mainly qualitative methods approach, specifically cross and descriptive with few participants and use usually only one tool in data collection, the interview. With regard to the matters investigated, most of the work is on the 'resilience and adversity' $(n=38 ; 42,7 \%)$. It was found
\end{abstract}


that resilience lacks an agreed definition and an established empirical tradition, emphasizing the importance of works like this to the researches.

Keywords: psychological resilience, scientific research.

\section{RESUMEN}

Los estudios acerca de la resiliencia psicológica han sido frecuentes en la producción científica brasileña. En general, las discusiones en este tema destacan los procesos de adaptación positivos como respuestas del individuo para afrontar adversidades significativas. Así, este estudio tiene como objetivo presentar una visión general de los trabajos científicos sobre la resiliencia psicológica en el ámbito nacional basada en el análisis de los artículos indexados en las bases de datos Lilacs y SciELO, desde 1970 hasta 2014. Han sido encontrados 89 artículos, de los cuales 47 son estudios empíricos y 42 corresponden a investigación teórica, la mayoría se centra en la capacidad de resistencia individual $(n=75 ; 84,3 \%)$. Además, se observó que los estudios adoptan, con frecuencia, métodos cualitativos, específicamente, diseños transversales y descriptivos con pocos participantes, así como utilizan, por lo general, sólo una herramienta para la recolección de datos: la entrevista. Con respecto a los temas investigados, la mayor parte de los trabajos tratan de la 'resiliencia y la adversidad' $(n=38$; $42,7 \%$ ). Se observó que la resiliencia carece de una definición acordada y una tradición empírica establecida, destacando la importancia de trabajos como este para los investigadores del tema.

Palabras clave: resiliencia psicológica, investigación científica.

\section{Introdução}

Durante a década de 1970 as investigações acerca da resiliência psicológica ganharam maior atenção (Bhana \& Bachoo, 2011; Masten, 2001), porém os debates sobre o assunto em congressos científicos são mais recentes, principalmente a partir do final dos anos noventa do século XX (Yunes, 2003). Verifica-se que desde os trabalhos pioneiros, até os mais atuais que investigam este construto, trazem que a resiliência ainda não apresenta uma definição consensual nem uma tradição empírica consistente (Cecconello, 2003; Junqueira \& Deslandes, 2003; Masten, 2001; Masten \& Reed, 2002; Poletto \& Koller, 2006; Poletto, Wagner, \& Koller, 2004; Rutter, 1993; Yunes, 2001, 2003). Em geral, as discussões a respeito do conceito de resiliência salientam os processos de adaptação positiva como respostas no enfrentamento de adversidades significativas (Bhana \& Bachoo, 2011; Fourie \& Theron, 2012; Masten, 2001, 2007; Masten \& Reed, 2002; Poletto \& Koller, 2008; Reppold, Mayer, Almeida, \& Hutz, 2012; Rolland \& Walsh, 2006; Rutter, 2012, 2013; Yunes \& Szymanski, 2001).

Historicamente, o estudo da resiliência pode ser dividido em dois focos: o individual e o familiar. Dentre a maioria das pesquisas sob o foco individual, as crianças ou adolescentes são estudados numa perspectiva em que traços e disposições pessoais são a chave para 0 desenvolvimento da resiliência (Yunes, 2003). De acordo com o foco 
familiar, há uma mudança no direcionamento dos estudos e no pensamento dos estudiosos ao se considerar o âmbito processual e relacional da resiliência. Desse modo, ressalta-se a possibilidade de desenvolvimento deste fenômeno, por meio do relacionamento com pessoas significativas, que estão presentes no entorno dos sujeitos, enquanto em situação de risco (Masten \& Obradović, 2006; Rutter, 1993; Ungar, 2011), podendo se destacar a resiliência em famílias.

Sob o panorama atual das pesquisas em desenvolvimento humano e à luz dos pressupostos da promoção e prevenção de saúde, estudos acerca da resiliência vêm sendo cada vez mais frequentes, porém com a utilização de instrumentos diversos. Tal variabilidade na metodologia sugere uma falta de consenso em torno do conceito do construto, convergindo com o que Yunes e Szymanski (2005) retratam como, "nós conceituais e metodológicos", os quais merecem destaque no presente artigo. Assim, apesar de Luthar, Cicchetti e Becker (2000) reiterarem que a variabilidade na utilização dos métodos é de extrema importância para a expansão da compreensão de qualquer construto científico, não há consenso sobre a melhor maneira de mensurar ou avaliar aspectos relacionados à resiliência (Lopes \& Martins, 2011; Luthar et al., 2000; Masten \& Obradović, 2006; Reppold et al., 2012).

Nesse contexto, a investigação de Oliveira, Reis, Zanelato e Neme (2008) sobre a metodologia empregada nos estudos acerca da resiliência no período de 2000 a 2006, sinaliza o uso prevalente de entrevistas $(38,09 \%)$ e a tendência no uso de escalas $(18,75 \%)$, bem como o de entrevistas ou de escalas combinadas com outros instrumentos (25\%). Os demais instrumentos utilizados nos estudos são questionários, fotografias, artesanatos, registro de campo e desenho, os quais totalizam $18,16 \%$. Em um estudo mais recente sobre resiliência familiar, Rooke e Pereira-Silva (2012) encontraram resultados similares aos de Oliveira et al., apontando a entrevista como o instrumento mais frequentemente utilizado (19\%), tanto no contexto nacional quanto no estrangeiro. O uso de questionário $(11,4 \%)$, bem como de narrativas $(3,8 \%)$ também ocorreu, porém em uma frequência menor.

De acordo com Cicchetti e Garmezy (1993) e Libório, Castro e Coêlho (2006) dependendo de como é feita a conceituação da resiliência, diferentes métodos de análise podem ser utilizados. Nesse sentido, a partir de uma clara definição teórica acerca do construto investigado, ter-se-á uma melhor comunicação entre os estudiosos, o que promoverá um consenso e favorecerá um fortalecimento da área de estudo com fundamentação metodológica e resultados mais consistentes (Cicchetti \& Garmezy, 1993). Logo, é fundamental que os estudos deixem clara a definição utilizada.

No que tange à viabilidade da mensuração da resiliência na realidade brasileira, observam-se tendências distintas. Nesse sentido, alguns 
pesquisadores (como, Lopes \& Martins, 2011; Pesce et al., 2005) realizaram adaptações transculturais e estudos de evidências de validade para escalas de resiliência que avaliam níveis deste construto. Em contraponto, Yunes (2001) salienta que o uso de escalas ou qualquer outro teste psicométrico contribui para a compreensão de indivíduos "resilientes" e "não resilientes", o que não retrata a complexidade do fenômeno.

Tendo em vista estes aspectos mencionados, o presente trabalho, além de contribuir no estudo e compreensão do construto, objetiva apresentar um panorama geral a respeito da produção científica na área de resiliência no âmbito nacional, a partir da análise de publicações acadêmicas entre 1970 e 2014, tendo como foco os tipos de publicação, a metodologia empregada, os temas mencionados, 0 tamanho da amostra e instrumentos/técnicas empregados para a coleta de dados.

\section{Método}

Foi realizada uma pesquisa de caráter bibliográfico, mediante a busca eletrônica de artigos nas bases de dados Lilacs e SciELO, abrangendo o período de 1970 a 2014. A escolha das bases foi feita, prioritariamente, por estas armazenarem uma grande quantidade de publicações acadêmicas nacionais, o que está concatenado com os objetivos do presente estudo. Focalizou-se a produção a partir da década de 1970 por este período representar a época em que as investigações acerca da resiliência psicológica adquiriram maior foco no meio científico (Bhana \& Bachoo, 2011; Masten, 2001).

A busca em cada uma das referidas bases foi norteada pelos descritores "resiliência" e "resiliência psicológica". Como resultados de busca foram encontradas 490 publicações nessas bases e, a partir destas, leituras prévias foram realizadas nos documentos para que houvesse uma seleção dos artigos. Para tal, alguns critérios de inclusão foram adotados. São eles: 1) os descritores "resiliência" e "resiliência psicológia" deveriam estar presentes no título do trabalho e no objetivo. Este critério foi utilizado para garantir que os estudos tivessem efetivamente a resiliência como foco e, não apenas, como um aspecto derivado da análise dos dados; 2) tratar-se de artigos indexados em periódicos, pois tais publicações são submetidas a um processo de avaliação cega por pares, o qual pode ser considerado um método amplamente aceito de garantia de qualidade do conhecimento produzido (Scorsolini-Comin \& Amorin, 2008).

Após a aplicação dos critérios de elegibilidade, resultaram 89 artigos, os quais foram lidos integralmente e analisados sistematicamente segundo as seguintes informações: 1) tipo de publicação: trabalhos empíricos ou pesquisas teóricas; 2) métodos e delineamentos dos 
estudos; 3) ano da publicação; 4) tipo de resiliência investigada: individual ou familiar; 5) assunto investigado; 6) tratando-se de trabalho empírico, tamanho da amostra, caracterização dos participantes e instrumentos/técnicas de coleta de dados.

No que tange aos temas investigados, foi realizada análise de conteúdo (Bardin, 1977/2011) para a construção das categorias (assuntos) e subcategorias de assuntos, sendo estas geradas a partir da análise dos assuntos contidos em cada trabalho. Ressalta-se que os dados coletados foram organizados em uma planilha do software Excel, visando facilitar a identificação e a análise.

\section{Resultados}

Foi encontrado um total de 89 trabalhos científicos sobre o tema resiliência. Destes, os trabalhos empíricos são mais frequentes $(n=47 ; 52,8 \%)$, seguidos por pesquisas teóricas $(n=42 ; 47,2 \%)$. No que concerne à utilização de métodos e delineamentos das investigações, a Tabela 1 apresenta sua categorização. Ressalta-se que esta classificação foi realizada a partir das especificações de Baptista e Campos (2007), nas quais são descritos o método quantitativo e qualitativo, bem como seus respectivos delineamentos. De forma geral, o método quantitativo abrange os estudos nos quais os dados são coletados, processados e analisados quantitativamente, enquanto no método qualitativo a subjetividade é reconhecida como fonte de informação, sendo o pesquisador responsável por retratar sua individualidade na pesquisa (Baptista \& Campos, 2007). Ainda de acordo com estes autores (Baptista \& Campos, 2007), a pesquisa quantitativa pode se caracterizar com diferentes delineamentos, por exemplo, correlacional (objetiva explorar relações que possam existir entre variáveis, exceto a relação de causa-efeito), validação (objetiva verificar se um teste mede aquilo que se propõe a medir) e estudo de coorte (objetiva analisar a relação existente entre a presença de fatores de riscos e o desenvolvimento de enfermidades nos grupos estudados). Já as pesquisas qualitativas englobam os delineamentos descritivos (objetivam descrever os dados coletados), delineamentos fenomenológicos (objetivam descrever a historicidade dos fenômenos individuais ou grupais, sendo o fenômeno a ser pesquisado o ponto de partida do conhecimento) e estudos de caso (objetivam adquirir um conhecimento amplo e detalhado de um indivíduo ou grupo a partir do estudo dos mesmos) (Baptista \& Campos, 2007). Destacamse ainda os estudos transversais, aqueles em que os dados são coletados em um só momento e os estudos longitudinais, aqueles em que os dados são coletados ao longo do tempo.

Como resultados, verifica-se a predominância de estudos brasileiros que adotam métodos qualitativos, especificamente, delineamentos 
transversais e descritivos na execução de pesquisas com foco na resiliência.

Tabela 1

Métodos e delineamentos dos estudos empíricos

\begin{tabular}{cccc}
\hline \multirow{3}{*}{ Delineamentos } & Métodos Qualitativos & $\mathbf{N}$ & $\%$ \\
& Descritivo/Transversal & 19 & 61,3 \\
& Estudo de caso/Transversal & 11 & 35,5 \\
& Fenomenológico/Transversal & 1 & 3,2 \\
\hline Total & & 31 & 100,0 \\
\hline \multirow{3}{*}{ Delineamentos } & Métodos Quantitativos & & \\
& Correlacional/Transversal & 12 & 75 \\
& Validação/Transversal & 2 & 12,5 \\
& Correlacional/Longitudinal & 1 & 6,25 \\
& Estudo de & 1 & 6,25 \\
& coorte/Longitudinal & & \\
\hline Total & & 16 & 100,0 \\
\hline
\end{tabular}

Em relação à distribuição das investigações ao longo dos anos, a Tabela 2 apresenta sua disposição. Constata-se que, ao longo dos anos, há um aumento da produção, especialmente, a partir do ano 2009. Os anos $2011(n=13 ; 14,6 \%)$ e $2012(n=13 ; 14,6 \%)$ foram aqueles com maior frequência de trabalhos publicados.

Tabela 2

Distribuição das investigações ao longo dos anos

\begin{tabular}{lcc}
\hline Ano & N & \% \\
\hline 1996 & 1 & 1,1 \\
1998 & 1 & 1,1 \\
1999 & 2 & 2,2 \\
2001 & 2 & 2,2 \\
2002 & 1 & 1,1 \\
2003 & 4 & 4,5 \\
2004 & 3 & 3,4 \\
2005 & 6 & 6,8 \\
2006 & 7 & 7,9 \\
2007 & 4 & 4,5 \\
2008 & 4 & 4,5 \\
2009 & 8 & 9 \\
2010 & 11 & 12,4 \\
2011 & 13 & 14,6 \\
2012 & 13 & 14,6 \\
2013 & 9 & 10,1 \\
\hline Total & 89 & 100,0 \\
\hline
\end{tabular}

No que tange ao tipo de resiliência pesquisada, a maioria dos estudos refere-se à resiliência individual $(n=75 ; 84,3 \%)$. Dentre estes, os estudos empíricos são mais freqüentes $(n=41 ; 54,7 \%)$, seguidos 
pelos teóricos $(n=34 ; 45,3 \%)$. A resiliência familiar é foco de apenas 10 trabalhos $(15,7 \%)$, sendo 6 empíricos $(60 \%)$ e 4 teóricos $(40 \%)$. Quatro investigações retratam tanto a resiliência individual quanto a familiar, sendo todas teóricas.

\subsection{Assuntos investigados nos trabalhos científicos}

Os trabalhos encontrados foram agrupados em três assuntos investigados, 'resiliência e adversidades', 'discussão acerca da resiliência' e 'resiliência e promoção de saúde'. A categoria 'resiliência e adversidades' abarca os trabalhos que tratam do desenvolvimento da resiliência através de algum evento adverso ou desfavorável. Nesta perspectiva, algumas subcategorias contemplam esta categoria como, 'Doenças/Deficiências', 'Baixa renda', 'Violência', 'Situação de rua' (refere-se aos moradores de rua), 'Institucionalização' (refere-se aos moradores de orfanatos) e 'Encarceramento' (refere-se aos presos). Em relação à categoria 'discussão acerca da resiliência', foram incluídos os artigos que tinham como temática aspectos sobre o fenômeno da resiliência, sejam conceituais ou metodológicos. Três subcategorias compõem esta categoria: 'Análise da produção científica', 'Mensuração/Validação de escalas' e 'Contribuição de teorias' (refere-se aos artigos que tratam da contribuição de teorias distintas para a compreensão da resiliência). Já na categoria 'resiliência e promoção de saúde' foram considerados os trabalhos que articulavam o estudo e desenvolvimento da resiliência com estratégias relacionadas à redução de situações de vulnerabilidade e assim, melhoria da qualidade de vida. Duas subcategorias contemplam esta categoria, 'Construtos convergentes' e 'Políticas públicas'. A primeira refere-se aos trabalhos que sistematizam 0 construto da resiliência com outros que favorecem o seu desenvolvimento como, fatores de proteção, estratégias de coping, investigando suas interfaces com a promoção da saúde. Posteriormente, 'Políticas públicas' trata dos artigos que associam o estudo e compreensão do processo da resiliência para a construção de políticas públicas com base na promoção de saúde.

No que tange às frequências das categorias investigadas nos trabalhos científicos, a maioria refere-se à 'resiliência e adversidades' $(n=38 ; 42,7 \%)$, seguidos por estudos que focalizam a 'discussão acerca da resiliência' $(n=35 ; 39,3 \%)$ e 'resiliência e promoção de saúde' $(n=16 ; 18 \%)$.

Cada investigação, de acordo com a categoria geral dos assuntos acima mencionada, foi analisada segundo as subcategorias de assuntos. Na categoria 'resiliência e adversidades', o assunto mais frequente refere-se a 'doenças/deficiências' $(n=11 ; 12,4 \%)$, seguido por 'baixa renda' $(n=9 ; 10,1 \%)$ e 'adversidades não especificadas' $(\mathrm{n}=8 ; 9 \%)$. Já na categoria 'discussão acerca da resiliência', há 
predominância da subcategoria 'análise da produção científica' $(n=21 ; 23,6 \%)$. A subcategoria 'construtos convergentes' $(n=14$; $15,8 \%$ ) é mais frequentemente investigada na categoria 'resiliência e promoção de saúde'. A Tabela 3 apresenta as subcategorias considerando cada categoria geral de assuntos.

Tabela 3

\begin{tabular}{lcc} 
Percentual de Subcategorias de Assuntos Investigados \\
\hline Subcategorias de assuntos & N & $\%$ \\
\hline Resiliência e adversidades & 11 & 12,4 \\
Doenças/Deficiências & 9 & 10,1 \\
Baixa renda & 8 & 9 \\
Adversidades não especificadas & 4 & 4,5 \\
Violência & 3 & 3,4 \\
Situação de rua & 2 & 2,2 \\
Institucionalização & 1 & 1,1 \\
Encarceramento & 21 & 23,6 \\
\hline Discussão acerca da resiliência & 9 & 10,1 \\
Análise da produção científica & 5 & 5,6 \\
Contribuição de teorias & \multicolumn{3}{c}{} \\
Mensuração/Validação de escalas & 5 & 15,8 \\
\hline Resiliência e promoção de saúde & 2 & 2,2 \\
Construtos convergentes & 89 & 100 \\
Políticas públicas
\end{tabular}

\subsection{Tamanho e caracterização da amostra}

Para a caracterização e tamanho da amostra utilizada foram consultados apenas os trabalhos empíricos. Destaca-se que as informações dos artigos que focavam a resiliência individual foram separadas das dos artigos que se referiam à resiliência familiar para melhor compreensão acerca da amostra e do construto da resiliência. Em relação ao tamanho da amostra nos estudos sobre resiliência individual, foram identificados trabalhos que empregaram de 1 a 10 participantes $(n=16)$, de 10 a 20 participantes $(n=3)$, de 20 a 35 $(n=3)$, de 60 a $100(n=6)$, de 100 a $140(n=7)$, de 260 a $465(n=3)$, bem como aqueles com mais de 1000 participantes $(n=3)$. No que tange à faixa etária dos participantes, a maioria dos estudos utiliza adultos em sua amostra $(n=24)$, seguidos por participantes na adolescência $(n=10)$, na velhice $(n=4)$ e na infância $(n=3)$. Ressaltase que esta classificação das faixas etárias foi realizada de acordo com o Estatuto da Criança e do Adolescente (Brasil, 1990) e o Estatuto do Idoso (Brasil, 2003). Já o tamanho da amostra das investigações sobre resiliência familiar variou entre uma família $(n=1)$, duas famílias $(n=2)$, quatro famílias $(n=1)$, doze famílias $(n=1)$ e trinta e duas famílias $(n=1)$. 


\subsection{Instrumentos/técnicas de coleta de dados}

Para a especificação dos instrumentos e técnicas de coleta de dados foram consultados novamente apenas os trabalhos empíricos. Destaca-se que os artigos acerca da resiliência individual e familiar foram analisados separadamente para melhor compreensão acerca do construto da resiliência. Nos estudos que focalizam a resiliência individual, verifica-se que a maioria utiliza três ou mais instrumentos/técnicas $(n=17 ; 41,5 \%)$, seguida pela utilização de apenas um(a) instrumento/técnica $(n=16 ; 39 \%)$. O uso de dois instrumentos é verificado em oito estudos (19,5\%). No que concerne às pesquisas acerca da resiliência familiar, há predominância da utilização de apenas uma técnica $(n=4 ; 66,6 \%)$, seguido pelo uso de três ou mais instrumentos/técnicas $(n=2 ; 33,4 \%)$. Conforme a Tabela 4 apresenta, houve uma grande variedade nos tipos de instrumentos/técnicas utilizados pelas investigações.

Tabela 4

Instrumentos/técnicas de coleta de dados

\begin{tabular}{|c|c|c|c|}
\hline & $\begin{array}{l}\text { Resiliência Individual } \\
\text { Entrevista }\end{array}$ & $\begin{array}{c}\mathbf{N} \\
12\end{array}$ & $\begin{array}{c}\% \\
29.3\end{array}$ \\
\hline & Escala de Resiliência & 3 & 7,3 \\
\hline & Narrativa & 1 & 2,4 \\
\hline & $\begin{array}{l}\text { Escala de Resiliência e } \\
\text { questionário }\end{array}$ & 5 & 12,2 \\
\hline \multirow[t]{8}{*}{ Instrumentos/técnicas } & Observação e entrevista & 2 & 4,9 \\
\hline & Escala de Resiliência e teste & 1 & 2,4 \\
\hline & $\begin{array}{l}\text { Escala de Resiliência, teste e } \\
\text { escala }\end{array}$ & 12 & 29,3 \\
\hline & $\begin{array}{l}\text { Escala de Resiliência, } \\
\text { questionário e teste }\end{array}$ & 3 & 7,3 \\
\hline & $\begin{array}{l}\text { Entrevista, questionário e } \\
\text { teste }\end{array}$ & 2 & 4,9 \\
\hline & Total & 41 & 100,0 \\
\hline & Resiliência Familiar & & \\
\hline & Entrevista & 4 & 66,6 \\
\hline \multirow[t]{3}{*}{ Instrumentos/técnicas } & $\begin{array}{l}\text { Entrevista, observaçåo e } \\
\text { questionário }\end{array}$ & 1 & 16,7 \\
\hline & $\begin{array}{l}\text { Entrevista, genograma e } \\
\text { questionário }\end{array}$ & 1 & 16,7 \\
\hline & Total & 6 & 100,0 \\
\hline
\end{tabular}

\section{Discussão}


De modo geral, apesar da busca ter sido realizada apenas em periódicos, não abrangendo, portanto, livros, capítulos de livros, dissertações ou teses, obteve-se um panorama geral das investigações acerca da resiliência no Brasil. Assim, verifica-se uma escassez de trabalhos na área com números praticamente equivalentes de investigações empíricas e teóricas. Tais resultados sugerem que muitos pesquisadores ainda não têm clareza quanto ao conceito e características do construto, justificando o alto índice de trabalhos teóricos. Consequentemente a esta realidade, ao realizar pesquisas empíricas sobre o tema, estudiosos experienciam dúvidas e incertezas no que tange à fundamentação metodológica, o que pode comprometer os resultados apresentados.

Em relação aos anos de publicações com o foco na resiliência, o artigo mais antigo encontrado data de 1996, corroborando com Yunes (2003) quando a autora salienta que os debates acerca do fenômeno psicológico iniciaram em congressos científicos principalmente a partir do final dos anos noventa do século XX.

No que concerne ao método utilizado nos estudos, constatou-se que a maioria das pesquisas empíricas nacionais acerca da resiliência adota uma análise qualitativa com predomínio da utilização de delineamentos descritivos. Segundo Libório et al. (2006), a preocupação mais recente dos pesquisadores é entender como variáveis como fatores de risco e de proteção podem contribuir para um resultado positivo ou não e quais são os possíveis mecanismos mediadores dessa adaptação. Nesse sentido, a escolha do método qualitativo parece ser a mais adequada para as pesquisas acerca do construto.

Já as investigações quantitativas, na maioria das vezes, são estudos correlacionais, nos quais há uma tentativa de associar a resiliência a construtos convergentes (por exemplo, estratégias de coping). Tais investigações são, geralmente, aquelas com mais de vinte participantes e que utilizam três ou mais instrumentos como, a escala de resiliência, outras escalas e testes. No entanto, como Yunes (2001) afirma, um dos equívocos que se pode cometer nos estudos acerca deste tema e que é uma característica comum às teorias psicológicas, é o fato de se assumir uma perspectiva ideológica que rotule, classifique e estabilize determinados indivíduos ou populações, de forma binária e excludente, como resilientes ou não resilientes, 0 que não traduz a verdadeira essência do construto (Yunes, 2001).

A resiliência individual é foco predominante dos estudos brasileiros empíricos. Já a resiliência familiar é pouco investigada no campo teórico e empírico, sugerindo que as incertezas por parte dos estudiosos relacionadas a este construto podem ser mais complexas do que em relação ao primeiro. Além disso, os estudos acerca do tema "família" trazem, geralmente, discussões culpabilizando este sistema por problemas ou disfunções de alguns dos seus membros 
(Walsh, 1998/2005, 2002, 2003; Yunes, 2003; Yunes \& Szymanski, 2006).

Logo, a culpabilização do grupo familiar destacada nas pesquisas pode fazer com que os pesquisadores tenham dificuldades em ter um olhar mais positivo em relação ao grupo familiar e assim, realizar investigações sob o enfoque da resiliência em famílias.

Trabalhos que abarcam a resiliência individual e familiar ainda são mais raros e restringem-se a estudos teóricos. Nesse contexto, ressalta-se que a perspectiva desenvolvimental considera que a resiliência representa o sucesso das famílias, enquanto grupo, bem como depende do desenvolvimento de cada um dos seus membros (Masten \& Reed, 2002), porém questiona-se se a condição de uma destas "resiliências" implica, necessariamente, no desenvolvimento da outra. De acordo com Walsh (1998/2005), mais que um conjunto de traços determinados, o enfrentamento e a adaptação abrangem processos multideterminados que se estendem ao longo do tempo. A maioria das formas de estresse não é simplesmente um estímulo isolado, a curto prazo, mas um conjunto complexo de condições que mudam com uma história passada e um curso futuro (Rutter, 1987). Quanto aos assuntos investigados nos estudos, 'resiliência e adversidades' constitui a principal categoria encontrada, sendo mais frequente a subcategoria 'Doenças/deficiências'. Nesse sentido, apesar de, por muito tempo, as pesquisas sobre deficiência e doença indicarem um panorama desanimador, no qual havia a prevalência de estresse, depressão e isolamento social no sistema familiar (PereiraSilva \& Dessen, 2006; Povee, Roberts, Bourke, \& Leonard, 2012), atualmente os estudos têm reconhecido e indicado uma adaptação mais positiva tanto da pessoa com alguma doença ou deficiência quanto da família em relação a este membro (Blacher \& Baker, 2007; Fourie \& Theron, 2012; Risdal \& Singer, 2004; Van Riper, 2007), favorecendo, portanto, os estudos acerca da resiliência no contexto da deficiência e doença.

Outra subcategoria frequente nos trabalhos é 'Baixa renda'. De acordo com Rutter (2013), a pobreza e a desvantagem social têm efeitos robustos de risco na vida das pessoas. A privação econômica é, para a criança, fonte principal de risco sociocultural, sendo a pobreza e a miséria importantes fatores de risco universais (Poletto \& Koller, 2006). Assim, os estudos encontrados destacam algumas pessoas ou famílias que através da pobreza desenvolveram 0 processo de resiliência. Estes resultados evidenciam a necessidade de investigações sobre $o$ construto da resiliência para que novos conhecimentos embasem a realização de políticas públicas, especialmente para as pessoas com baixo status socioeconômico já que, no Brasil, a posição socioeconômica é um fator importante para a configuração do acesso da família a recursos fundamentais, como a manutenção da saúde e do bem-estar do grupo familiar. Todavia, 
ressalta-se que conforme Poletto et al. (2004) apontam, a resiliência representa um novo olhar diante das adversidades, mas não deve ser considerada como uma saída mágica.

A segunda categoria mais frequente é 'Discussão acerca da resiliência', tendo a subcategoria 'Análise da produção científica' presente em muitos estudos. Estes resultados podem ser justificados, provavelmente, por muitos trabalhos, pioneiros ou atuais, retratarem que a resiliência ainda não apresenta uma definição consensual nem uma tradição empírica consistente (Cecconello, 2003; Junqueira \& Deslandes, 2003; Masten, 2001; Masten \& Reed, 2002; Poletto \& Koller, 2006; Poletto et al., 2004; Rutter, 1993; Yunes, 2001, 2003). A categoria menos frequente é 'Resiliência e promoção de saúde', porém destaca-se que nesta houve predomínio da subcategoria 'Construtos convergentes'. Além de não dispor de uma conformidade conceitual e uma fundamentação metodológica, uma multiplicidade de fatores e variáveis devem ser considerados no estudo dos fenômenos humanos como a resiliência (Poletto \& Koller, 2006; Yunes, 2003). Dessa maneira, provavelmente, os estudiosos tendem a pesquisar a resiliência e outros construtos convergentes para que, assim, os dados coletados possam se complementar, tornando os resultados mais confiáveis e consistentes.

No que se refere ao tamanho da amostra, os estudos com foco na resiliência individual e familiar utilizam poucos participantes, o que está coerente com a escolha do método, visto que há predominância de trabalhos que adotam o método qualitativo e este não se caracterizar por uma grande amostra. De acordo com Denzin e Lincoln (2006), em estudos qualitativos, a seleção de participantes privilegia a sua importância para o tema investigado ao invés da sua representatividade. Assim, a generalização não representa uma preocupação ou um objetivo para estes estudos. No entanto, verificase que os trabalhos acerca da resiliência individual apresentaram variedade no número de participantes. Geralmente, as investigações com um a dez participantes são estudos de caso e os com mais de mil, estudos de validação de escalas de resiliência.

Em relação à faixa etária dos participantes dos estudos acerca da resiliência individual, destaca-se que apesar dos adultos constituírem a principal amostra encontrada, a resiliência pode ser desenvolvida em qualquer momento ao longo de todo o ciclo de vida e neste sentido, nenhuma fase do desenvolvimento deve ser privilegiada nas investigações. Walsh (1998/2005) afirma que a compreensão de ciclo de vida sobre o desenvolvimento individual e familiar é necessária para o entendimento da resiliência já que esta não deve ser avaliada somente uma vez através de uma captação rápida das interações ocorridas. A autora enfatiza que, dessa maneira, pode-se deixar de apreciar que as pessoas estão se desenvolvendo e têm trajetórias flexíveis e multideterminadas durante a vida, negando a natureza 
dinâmica da resiliência. Assim, reitera-se a necessidade de estudos longitudinais na área para que o processo de variabilidade da resiliência, bem como suas características, possam ser compreendidos de forma mais fundamentada e consistente pelos estudiosos.

Por último, as pesquisas acerca da resiliência individual utilizam, frequentemente, três ou mais instrumentos/técnicas, sendo um destes uma escala de resiliência. Destaca-se que nos trabalhos encontrados, duas escalas de resiliência foram frequentemente utilizadas: escala de resiliência, desenvolvida em 1993 por Wagnild e Young e escala de resiliência de Connor-Davidson (Cd-Risc-10). Nesse contexto, destaca-se a importância do pluralismo metodológico, em especial para as investigações acerca de "novos construtos" como a resiliência individual e familiar, as quais ainda carecem de uma tradição empírica consolidada. Portanto, novos e distintos conhecimentos oriundos de diferentes instrumentos de coleta de dados, tais como questionários, escalas, inventários, testes e entrevistas são muito bem-vindos para os pesquisadores da área, pois favorecem o desenvolvimento e aprimoramento do tema.

Contudo, para Yunes e Szymanski (2005) o uso de instrumentos quantificadores de resiliência não é adequado, pois estes, geralmente, medem o inquantificável, desconsiderando os fatores que ocorrem em uma situação natural com as pessoas e retratando somente o que é reportado nos instrumentos como os riscos definidos a priori. Assim, segundo Reppold et al. (2012), Yunes (2001) e Yunes e Szymanski (2001) as tendências para a pesquisa em resiliência ressaltam a importância da subjetividade dada pelo sujeito acerca das adversidades. Para Libório et al. (2006) a ideia de condições universais apriorísticas do que são condições de vulnerabilidade e do que serve como risco ou proteção deve ser abolida em prol da contextualização do que as pessoas consideram como ameaça ou não.

Ainda sob a perspectiva destes autores (Libório et al., 2006), por se tratar de um fenômeno, sugere-se não utilizar o termo resiliente como uma qualidade ou um adjetivo de indivíduos, pois, assim, tratase o construto como uma qualidade imutável, um traço, o que favorece a compreensão de uma pessoa invulnerável e distancia-se do desenvolvimento de políticas públicas e institucionais preventivas. Todavia, algumas pesquisas nacionais ainda trazem em seus resultados a expressão "resiliente", não retratando com cuidado nem com cautela suas afirmações e conclusões. Logo, acredita-se que com os avanços das pesquisas na área do desenvolvimento humano, seja mais adequada a utilização de uma definição que considere a resiliência como um processo. Além disso, face à complexidade do fenômeno e à sua natureza dinâmica e contextual, a avaliação da resiliência não pode ser fundamentada em um instrumento único ou 
unidimensional, ou como uma condição estável do sujeito, devendo ser relativizada em relação aos fatores de risco e proteção do contexto (Reppold et al., 2012).

Ainda com relação ao uso de instrumentos nos estudos analisados, a utilização de um só instrumento também foi frequente nos trabalhos com foco na resiliência individual e familiar. Destaca-se que em ambos os estudos, o instrumento mais utilizado para investigar a resiliência é a entrevista, corroborando com os resultados apontados por Oliveira et al. (2008) e Rooke e Pereira-Silva (2012). Além disso, como já mencionado, o presente artigo constatou que a maioria das investigações nacionais acerca do construto é qualitativa, o que justifica a tendência ao uso de entrevistas pelos pesquisadores.

Concebendo a tamanha complexidade e o não esgotamento do assunto acredita-se ter suscitado novas reflexões, questionamentos e indagações, fomentando a produção teórica e empírica sobre a resiliência individual e familiar. Espera-se que, assim, haja um crescente número de pesquisas na área, englobando distintos participantes em diferentes fases do desenvolvimento, bem como uma variedade de arranjos familiares, ambos em diferentes contextos e condições. Além disso, almeja-se que estudos descritivos e explicativos embasem a elaboração de projetos e programas de intervenção e que as políticas públicas vinculem a prática com as pessoas, famílias e comunidades às descobertas acadêmicas mais recentes.

\section{Referências}

Bardin, L. (1977/2011). Análise de conteúdo. Lisboa: Edições 70.

Baptista, M. N., \& Campos, D. C. (2007). Metodologias de pesquisa em ciências: análises quantitativa e qualitativa. Rio de Janeiro: LTC.

Bhana, A., \& Bachoo, S. (2011). The determinants of family resilience among families in low-and-middle-income contexts: a systematic literature review. South African Journal of Psychology, 41, 131-139.

Blacher, J., \& Baker, B. L. (2007). Positive impact of intellectual disability on families. American Journal on Mental Retardation, $112,330-348$.

Brasil (1990). Lei n. 8.063, de 13 de julho de 1990. Estatuto da Criança e do Adolescente (ECA). Recuperado de http://portal.mec.gov.br/seesp/arquivos/pdf/lei8069_02.pdf

Brasil (2003). Lei n. 10.741, de 10 de outubro de 2003. Estatuto do Idoso. Recuperado de http://www.planalto.gov.br/ccivil_03/leis/2003/l10.741.htm 
Cecconello, A. M. (2003). Resiliência e vulnerabilidade em famílias em situação de risco (Tese de Doutorado, Universidade Federal do Rio Grande do Sul, Porto Alegre).

Cicchetti, D., \& Garmezy, N. (1993). Milestones in the development of resilience. Development and Psychopathology, 5, 497-774.

Denzin, N. K., \& Lincoln, Y. S. (2006). O planejamento da Pesquisa Qualitativa. Porto Alegre: Artmed Bookman.

Fourie, C. L., \& Theron, L. C. (2012). Resilience in the face of fragile X syndrome. Qualitative Health Research, 22, 1355-1368.

Junqueira, M. F. P. S., \& Deslandes, S. F. (2003). Resiliência e maus tratos à criança. Cadernos de Saúde Pública, 19, 227-235.

Libório, R. M. C., Castro, B. M., \& Coêlho, A. E. L. (2006). Desafios metodológicos para a pesquisa em resiliência: conceitos e reflexões críticas. In D. D. Dell'Aglio, S. H. Koller, \& M. A. M. Yunes (Orgs.), Resiliência e Psicologia Positiva: Interfaces do Risco à Proteção (pp. 89-115). São Paulo: Casa do Psicólogo.

Lopes, V. R., \& Martins, M. C. F. (2011). Validação fatorial da escala de resiliência de Connor-Davidson (Cd-Risc-10) para brasileiros. Revista Psicologia: Organizações e Trabalho, 11, 36-50.

Luthar, S. S., Cicchetti, D., \& Becker, B. (2000). The construct of resilience: a critical evaluation and guidelines for future work. Child Development, 71, 543-562.

Masten, A. S. (2001). Ordinary magic: resilience processes in development. American Psychologist, 56, 227-238.

Masten, A. S. (2007). Resilience in developing systems: progress and promise as the fourth wave rises. Development and Psychopathology, 19, 921-930.

Masten, A. S., \& Obradović, J. (2006). Competence and resilience in development. Annals New York Academy of Sciences, 1094, 1327.

Masten, A. S., \& Reed, M. G. (2002). Resilience in development. In C.R. Snyder \& S.J. Lopez (Eds.), The handbook of positive psychology (pp. 74-88). New York: Oxford University Press.

Oliveira, M. A., Reis, V. L., Zanelato, L. S., \& Neme, C. M. B. (2008). Resiliência: análise das publicações no período de 2000 a 2006. Psicologia: Ciência e Profissão, 28, 754-767.

Pereira-Silva, N. L., \& Dessen, M. A. (2006). Famílias de crianças com síndrome de Down: sentimentos, modos de vida e estresse parental. Interação em Psicologia, 10, 183-194.

Pesce, R. P., Assis, S. G., Avanci, J. Q., Santos, N. C., Malaquias, J. V., \& Carvalhaes, R. (2005). Adaptação transcultural, confiabilidade e validade da escala de resiliência. Caderno de Saúde Pública, 21, 436-448.

Poletto, M., \& Koller, S. H. (2006). Resiliência: uma perspectiva conceitual e histórica. In: D. D. Dell'Aglio, S. H. Koller \& M. A. 
M. Yunes (Orgs.), Resiliência e psicologia positiva: interfaces do risco à proteção (pp. 19-44). São Paulo: Casa do Psicólogo.

Poletto, M., \& Koller, S. H. (2008). Contextos ecológicos: promotores de resiliência, fatores de risco e de proteção. Estudos de Psicologia, 25, 405-416.

Poletto, M., Wagner, T. M. C., \& Koller, S. H. (2004). Resiliência e desenvolvimento infantil de crianças que cuidam de crianças: uma visão em perspectiva. Psicologia: Teoria e Pesquisa, 20, 241-250.

Povee, K., Roberts, L., Bourke, J., \& Leonard, H. (2012). Family functioning in families with a child with Down syndrome: a mixed methods approach. Journal of Intellectual Disability Research, 56, 961-973.

Reppold, C. T., Mayer, J. C., Almeida, L. S., \& Hutz, C. S. (2012). Avaliação da resiliência: controvérsia em torno do uso das escalas. Psicologia: Reflexão e Crítica, 25, 248-255.

Risdal, D., \& Singer, G. H. (2004). Marital adjustment in parents of children with disabilities: a historical review and meta-analysis. Research and Practice for Persons with Severe Disabilities, 29, 95-103.

Rolland, J. S., \& Walsh, F. (2006). Facilitating family resilience with childhood illness and disability. Current Opinion in Pediatrics, 18, 527-538.

Rooke, M. I., \& Pereira-Silva, N. L. (2012). Resiliência familiar e desenvolvimento humano: análise da produção científica. Psicologia em Pesquisa, 6, 179-186.

Rutter, M. (1987). Psychosocial resilience and protective mechanisms. American Journal of Orthopsychiatry, 57, 316331.

Rutter, M. (1993). Resilience: some conceptual considerations. Journal of Adolescent Health, 14, 626-631.

Rutter, M. (2012). Resilience as a dynamic concept. Development and Psychopathology, 24, 335-344.

Rutter, M. (2013). Annual research review: resilience-clinical implications. Journal of Child Psychology and Psychiatry, 54, 474-487.

Scorsolini-Comin, F., \& Amorim, K. S. (2008). Corporeidade: uma revisão crítica da literatura científica. Psicologia em Revista, 14, 189-214.

Ungar, M. (2011). The social ecology of resilience: addressing contextual and cultural ambiguity of a nascent construct. American Journal of Orthopsychiatry, 81, 1-17.

Van Riper, M. (2007). Families of children with Down syndrome: responding to "a change in plans" with resilience. Journal of Pediatric Nursing, 22, 116-128. 
Walsh, F. (1998/2005). Fortalecendo a resiliência familiar (M. F. Lopes, Trad.). São Paulo: Roca.

Walsh, F. (2002). A family resilience framework: innovative practice applications. Family Relations, 51, 130-137.

Walsh, F. (2003). Family resilience: a framework for clinical practice. Family Process, 42, 1-18.

Yunes, M. A. M. (2001). A questão triplamente controvertida da resiliência em famílias de baixa renda (Tese de Doutorado, Universidade Católica de São Paulo, São Paulo).

Yunes, M. A. M. (2003). Psicologia positiva e resiliência: o foco no indivíduo e na família. Psicologia em Estudo, 8, 75-84.

Yunes, M. A. M., \& Szymanski, H. (2001). Resiliência: noção, conceitos afins e considerações críticas. In: J. Tavares (Org.), Resiliência e educação (pp.13-42). São Paulo: Cortez.

Yunes, M. A. M., \& Szymanski, H. (2005). Entrevista reflexiva \& grounded-theory: estratégias metodológicas para compreensão da resiliência em famílias. Revista Interamericana de Psicologia, 39, 431-438.

Yunes, M. A. M., \& Szymanski, H. (2006). O estudo de uma família que supera as adversidades da pobreza: um caso de resiliência em família. Psicodebate: Psicologia, Cultura y Sociedad, 7, 119139.

\section{Endereço para correspondência \\ Mayse Itagiba Rooke}

Universidade Federal de Juiz de Fora

Departamento de Psicologia, Instituto de Ciências Humanas

Rua José Lourenço Kelmer, CEP 36036-900, Juiz de Fora - MG, Brasil

Endereço eletrônico: mirpsi@gmail.com

Recebido em: 01/08/2014

Reformulado em: 22/02/2015

Aceito para publicação em: 16/05/2015

\section{Notas}

* Doutoranda em Psicologia pela Universidade Federal de Juiz de Fora. 